\title{
Mortalidade por transtornos falciformes no estado da Bahia no período de 2012 a 2016
}

\author{
Mortality due to sickle cell disorders in the state of Bahia from 2012 to 2016 \\ Mortalidad por trastornos falciformes en el estado de Bahía en el período de 2012 a 2016 \\ Débora Mendes Santana ${ }^{1}$, Ellen Karine Alves Reis ${ }^{1}$, Renata da Silva Santana ${ }^{1}$, Saiury Santana \\ Nascimento ${ }^{1}$, Josely Bruce dos Santos ${ }^{1 *}$, Claudenice Ferreira dos Santos ${ }^{1}$.
}

\section{RESUMO}

Objetivo: Caracterizar a mortalidade por transtornos falciformes no estado da Bahia, no período de 2012 a 2016. Métodos: Tratou-se de um estudo ecológico, do tipo série temporal, com a utilização do Sistema de Informação Geográfica (SIG), para georreferenciamento dos óbitos por transtornos falciformes nas nove macrorregiões de saúde do estado da Bahia. Resultados: Notou-se que as taxas de óbito por transtornos falciformes ocorreram em maior proporção na macrorregião Leste, com maior quantitativo de óbitos na faixa etária entre 30 a 39 anos, em homens negros, solteiros, com escolaridade entre fundamental I e Ensino Médio incompleto e maior ocorrência de óbito no ambiente hospitalar. Considerações Finais: Os transtornos falciformes podem comprometer a capacidade dos indivíduos enfrentarem seus problemas, bem como afetar, de forma negativa, o potencial para tornarem-se seres produtivos, ocasionando redução em sua qualidade de vida. O estudo contribuirá para reformular e direcionar políticas públicas e recursos para a assistência adequada aos portadores dos transtornos e do traço falciforme.

Palavras-chave: Doença falciforme, Mortalidade, Macrorregião de saúde.

\begin{abstract}
Objective: To characterize the mortality due to sickle-cell disorders in the state of Bahia, between 2012 and 2016. Methods: This is an ecological study, of the temporal series type, using the Geographic Information System (GIS) for georeferencing of deaths due to disorders in the nine macroregions of health in the state of Bahia. Results: It was observed that death rates due to sickle-cell disorders occurred in a greater proportion in the eastern macroregion, with a higher number of deaths in the age range between 30 and 39 years, in black men, single, with elementary and incomplete high school education and higher occurrence of death in the hospital setting. Final Considerations: Sickle cell disorders can compromise the ability of individuals to face their problems, as well as negatively affect the potential to become productive beings, causing a reduction in their quality of life. The study will contribute to reformulate and direct public policies and resources for adequate care for patients with disorders and sickle cell trait.
\end{abstract}

Keywords: Sickle cell disease, Mortality, Macroregion of health.

\section{RESUMEN}

Objetivo: Caracterizar la mortalidad por trastornos falciformes en el estado de Bahía en el período de 2012 y 2016. Métodos: Fue un estudio ecológico, del tipo de serie temporal, con utiliación del Sistema de Información Geográfica (SIG), para georreferenciación de muertes por transtornos falciformes en las nueve macrorregiones de salud del estado de Bahía. Resultados: Se observó que las tasas de mortalidad por trastornos falciformes ocurrieron en mayor proporción en la macrorregión oriental, con mayor número de muertes en el grupo de edad entre 30 y 39 años, en hombres negros, solteros, con escolaridad entre primaria I y bachillerato incompleto y mayor ocurrencia de muerte en el ambiente hospitalario. Consideraciones Finales: Los trastornos de células falciformes pueden comprometer la capacidad de los individuos para enfrentar sus problemas, así como afectar negativamente el potencial para convertirse en seres productivos, provocando una reducción en su calidad de vida. El estudio contribuirá a reformular y orientar las políticas públicas y los recursos para una adecuada atención a los portadores de los trastornos falciformes.

Palabras clave: Transtornos falciformes, Mortalidad, Macrorregión de salud.

${ }^{1}$ Centro Universitário Jorge Amado (UNIJORGE), Salvador - BA. *E-mail: joselybruce3@gmail.com 


\section{INTRODUÇÃO}

A Doença Falciforme (DF) é uma das patologias de origem genética mais frequentes mundialmente. Caracteriza-se por uma mutação, originando uma hemoglobina anormal $(\mathrm{HbS})$, responsável por modificar a morfologia das hemácias, transformando-as em forma de foice (BRASIL, 2015).

Há outras hemoglobinas anormais, como a C, D, E etc. As doenças falciformes mais comuns são a anemia falciforme (HbSS), a S/Beta talassemia ( $/$ /B tal.), as heterozigoses ( $\mathrm{Hb} \mathrm{SC}),(\mathrm{Hb} \mathrm{SD}),(\mathrm{Hb} \mathrm{SE})$, entre outras (BRASIL, 2015).

Essa alteração genética teve origem na África, mesmo antes dos africanos serem obrigados a mudaremse para o Brasil, tornando-se escravos (RAMOS JT, et al., 2015). A Bahia é o Estado com maior prevalência da DF, tendo um caso para cada 650 crianças nascidas vivas (BRASIL, 2014).

O Ministério da Saúde menciona que a incidência da doença ou transtornos falciformes ocorra entre $2 \%$ a $6 \%$ da população residente nas cinco regiões brasileiras, sendo a maioria formada por afrodescendentes (SILVA NCH, et al., 2017). O traço falciforme é muito presente no Brasil e afeta 6 a 10\% da população afrodescendente, em função da alta miscigenação. Os Estados Bahia e Rio de Janeiro apresentam maior prevalência do traço, sendo que a Bahia e Pernambuco são os principais representantes da Região Nordeste (BRASIL, 2014).

O diagnóstico da patologia é muito complexo, o que exige a descoberta precoce da doença. Os exames laboratoriais utilizados para a detecção dos transtornos falciformes são: hemograma, teste de falcização, teste de solubilidade, eletroforese de hemoglobina fetal e hemoglobina A2 etc. (SILVA NCH, et al., 2017).

As principais complicações são crises álgicas, infeções, icterícia, priapismo, sequestro esplênico, úlceras em membro inferior, dactilite, déficit de crescimento, anemia, complicações cardiorrespiratórias, insuficiência renal e acidentes vasculares encefálicos, comprometendo as funções de órgãos vitais e aumentado o risco de mortalidade (RAMOS JT, et al., 2015).

Dados fidedignos sobre a mortalidade e a letalidade por DF no Brasil ainda são escassos e diversos fatores contribuem para a permanência dessa situação: a ausência de informação nos atestados de óbito, a inexistência de cadastros informatizados em hemocentros e centros de referência, e indivíduos sem diagnóstico. Isso evidencia uma urgência na reformulação da rede de informação sobre os transtornos falciformes (BRASIL, 2015).

Elevadas taxas de letalidade por DF ou transtorno falciforme, associadas à mortalidade precoce da população jovem e à escassez de estudos sobre óbitos, direcionam para o maior interesse em pesquisar a mortalidade pela patologia (FIGUEIREDO AKB, 2014). Nesse sentido, faz-se necessária a atuação do profissional enfermeiro nas diversas áreas de trabalho: atenção básica, no aconselhamento genético, no acompanhamento da gestante no pré-natal, no puerpério, na triagem neonatal, na terapia de hemotransfusão, nas emergências clínicas etc.

As mudanças no padrão socioeconômico, nas alterações climáticas e nos cuidados à saúde colaboram para a diversificação das manifestações clínicas, porém não elucidam a complexidade da situação na qual vários indivíduos portadores da DF ou transtornos falciformes apresentam diferentes manifestações, sendo consideradas como assintomáticas, leves, incapacitantes e aquelas que ocasionam o óbito (SILVA NCH, et al., 2017).

Considerando a forte miscigenação da população brasileira, a elevada influência da etnia africana na Bahia, a escassez de estudos sobre a mortalidade por transtornos falciformes e a subnotificação do número de óbitos, ouve o interesse em pesquisar a mortalidade por DF ou transtornos falciformes nas nove macrorregiões de saúde do Estado da Bahia.

O estudo teve como objetivo caracterizar a mortalidade por transtorno falciforme na Bahia, entre o período de 2012 a 2016, incluindo o estudo do perfil epidemiológico da população de óbitos, através das variáveis faixa etária, sexo, cor/raça, estado civil, escolaridade e óbitos por local de ocorrência. 


\section{MÉTODOS}

Tratou-se de um estudo ecológico, do tipo série temporal, tendo como população indivíduos que foram a óbito, no período de 2012 a 2016, residentes no estado da Bahia. As nove macrorregiões estudadas foram Centro-Leste, Centro-Norte, Extremo-Sul, Leste, Nordeste, Norte, Oeste, Sudoeste e Sul, sendo utilizado o Sistema de Informação Geográfica (SIG) para georreferenciamento ou geoprocessamento dos óbitos por transtornos falciformes nessas macrorregiões.

As variáveis utilizadas neste estudo e disponíveis no Departamento de Informática do Sistema Único de Saúde do Brasil (DATASUS) foram: macrorregião de saúde, ocorrência, ano, faixa etária, cor/raça, sexo, estado civil, escolaridade e local de ocorrência; Categoria da Classificação Internacional de Doenças (CID10: D57-Transtornos Falciformes e Causa da Classificação Internacional de Doenças (CID-BR-10: 053Anemias).

A coleta de dados foi realizada utilizando o DATASUS, base de dados do Sistema Único de Saúde (SUS), e o Sistema de Informação sobre Mortalidade (SIM), considerando o período de estudo. Os óbitos foram coletados por ano, ocorrência, pesquisados com base no Capítulo III da lista de Classificação Internacional de Doenças (CID-10), correspondendo aos óbitos de transtornos falciformes a partir da faixa etária de 0 a 79 anos.

Os dados sobre os nascidos vivos foram coletados através do Sistema de Informações de Nascidos Vivos (SINASC), com o auxílio do Tabulador Genérico do DATASUS (TABNET), sendo útil na tabulação de dados e cruzamento de informações. Coletaram-se os dados sobre nascidos vivos na opção Estatísticas Vitais do DATASUS, pelo grupo nascidos vivos, por local de ocorrência, macrorregião e ano, para o cálculo da taxa de mortalidade, no período entre 2012 e 2016.

A estimativa da taxa de mortalidade por transtornos falciformes nas macrorregiões de saúde do estado da Bahia foi realizada através de fórmula, calculando-se o número de óbitos por transtornos falciformes, dividindo-se pelo número de nascidos vivos para cada ano e multiplicando-se por cem mil habitantes.

Calcularam-se as taxas de mortalidade para os anos de 2012, 2013, 2014, 2015 e 2016, sendo compiladas em tabelas e gráficos, para análise e discussão dos dados, os quais foram apresentados em forma de taxas de mortalidade, em números absolutos e em porcentagens. Em seguida, os dados foram processados e as tabelas elaboradas através do Microsoft Excel 2013, contribuindo para a adequada interpretação e realização do estudo.

Na elaboração dos mapas dos municípios, por macrorregião de saúde, para o georreferenciamento das taxas de mortalidade por transtornos falciformes no Estado da Bahia, de forma anual, no período de 2012 a 2016, utilizou-se o SIG. Os municípios e as macrorregiões de saúde compuseram as unidades espaciais para o georreferenciamento dos dados sobre mortalidade. A geolocalização do quantitativo de óbitos por transtornos falciformes, para o grupo das causas, foi realizada através do Software ArcGIS 10.1.

Utilizou-se o código do município para a correlação entre a tabela construída no Microsoft Excel 2013 e a malha digital (mapa) do Estado da Bahia, para posterior análise da distribuição das taxas de mortalidade nas macrorregiões de saúde do Estado da Bahia. Foram gerados mapa, gráfico e tabela, apresentando intervalos de classe abrangendo as taxas de mortalidade calculadas por ano e a taxa cumulativa para o período de 2012 a 2016 (GOIS EC, et al., 2019).

\section{RESULTADOS E DISCUSSÃO}

Os transtornos falciformes ou doença falciforme (DF) representam um grande problema de saúde pública na Bahia. A elevada população de afrodescendentes residentes no estado demanda por uma assistência especializada aos portadores dessa patologia. Entre as nove macrorregiões de saúde estudadas, verificouse que a região Leste apresentou o maior número de óbitos por nascidos vivos (219,9 óbitos por 100.000 nascidos vivos durante os anos de 2012 a 2016), conforme mostra a Figura 1. Essa condição sugere que Salvador, por ser a capital do estado da Bahia, detentora de um Hemocentro, além de unidades básicas de referência em transtornos falciformes e postos de saúde que fazem a coleta de sangue, tenha estrutura para diagnosticar e tratar os portadores da doença. 
Figura 1 - Distribuição espacial das taxas de mortalidade por transtornos falciformes segundo as macrorregiões de saúde no estado da Bahia no período de 2012 a 2016.

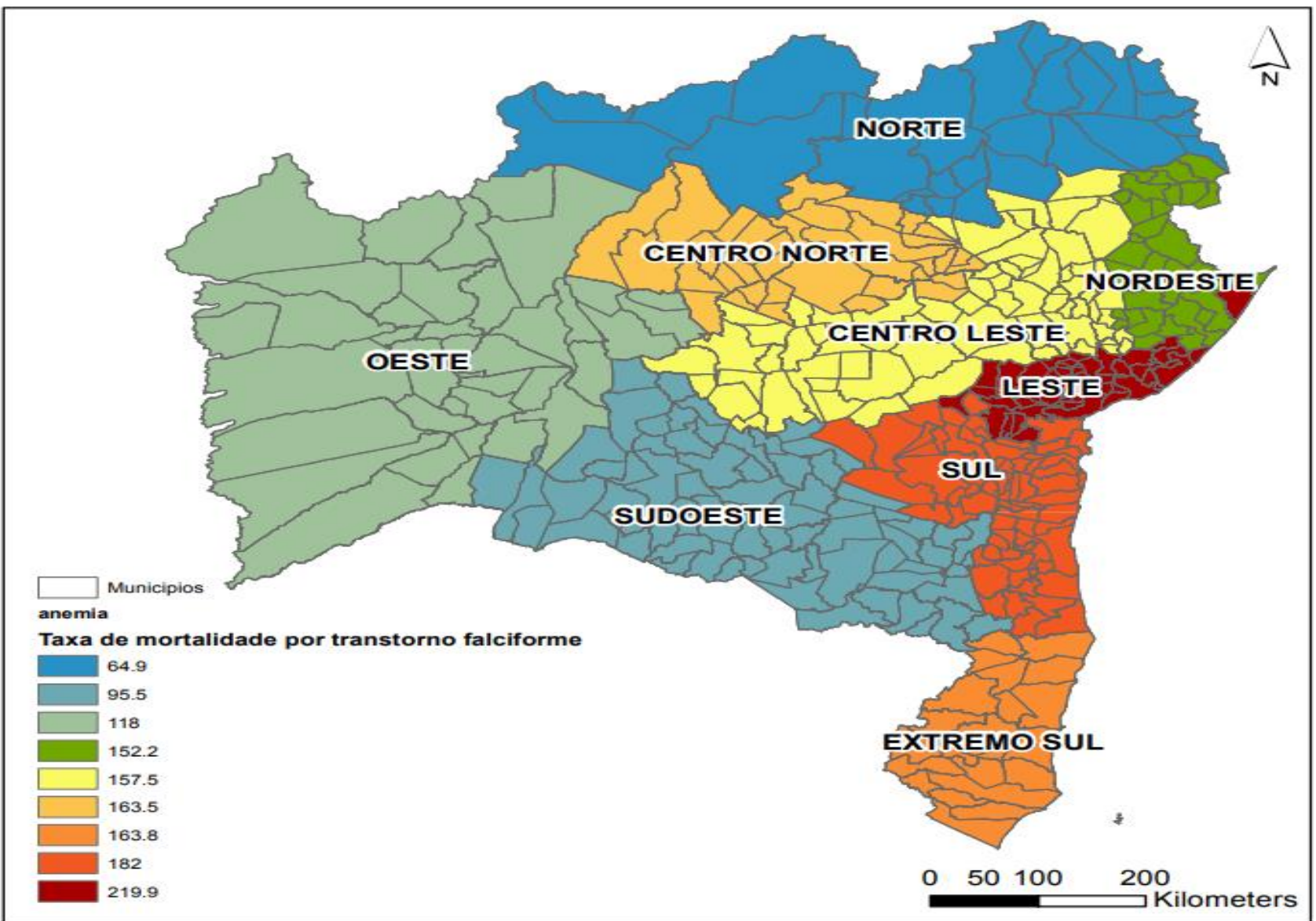

Fonte: Santana DM, et al., 2020. Dados coletados do Sistema de Informação Geográfica (SIG) (2018).

No ano de 2015, as taxas de óbito por transtornos falciformes corresponderam ao valor total de 32, o que representa o coeficiente de mortalidade de 49,8 para cada 100.000 nascidos vivos, com maiores taxas de óbitos na faixa etária entre 30 a 39 anos, em homens negros, solteiros, escolaridade entre fundamental I e Ensino Médio incompleto e maior ocorrência de óbito no âmbito hospitalar.

De acordo com o Ministério da Saúde, a genética para a doença falciforme é considerada um fator intrínseco aos indivíduos, não podendo ser alterada. No entanto, faz-se necessária uma assistência específica, durante o planejamento familiar, triagem neonatal, consulta de puericultura, saúde da criança e do adolescente e saúde do adulto, com o objetivo de melhorar a qualidade de vida dos portadores da doença falciforme (BRASIL, 2013).

Estudos relatam que a origem do transtorno falciforme no Brasil sucedeu com a imigração obrigatória de povos africanos-negros, para serem submetidos a trabalhos forçados no período da escravidão, sendo atualmente propagado em todo o mundo, atingindo predominantemente pessoas negras, pardas e pouco frequente em brancos. A população negra, a raça dominantemente acometida, está na base da pirâmide social e mostra os piores indicadores epidemiológicos, educacionais e econômicos (FIGUEIRÓ AVM e RIBEIRO RLR, 2012).

Independente da doença, o fator social é um componente crucial para a piora das manifestações clínicas. $\mathrm{Na}$ maior parte dos casos, os portadores do transtorno falciforme são pretos e pardos, com menor poder aquisitivo, baixa escolaridade, com limitações às comodidades dos serviços de saúde. Dados estatísticos revelam que esta população apresenta uma maior demanda pela assistência à saúde, devido a sua vulnerabilidade social (BRASIL, 2014). 
Nota-se que a fragilidade étnico-racial se apresenta por uma discriminação institucional que caracteriza o não comprometimento das instituições e organizações em manter um serviço com qualidade e humanização a esses indivíduos, em função de suas características, como origem racial ou étnica, cultura, dentre outros aspectos (FIGUEIRÓ AVM e RIBEIRO RLR, 2012).

A discriminação pela equipe multidisciplinar de saúde, a ausência de acesso aos serviços, o não direcionamento dos recursos financeiros nas políticas públicas da população negra, a negligência na gestão em educação continuada para os profissionais de saúde, são exemplos de racismo institucional no Sistema Único de Saúde (LAGES SRC, et al., 2017).

O preconceito racial é estruturante na desigualdade social brasileira, representando a forma como a sociedade o percebe na composição histórica e social, afetando, consideravelmente, as pessoas que são vítimas da discriminação racial e que convivem com os transtornos falciformes, um fator que as torna muito mais vulneráveis (OLIVEIRA AS e CARVALHO AR, 2017).

O pacto pela saúde buscou reduzir essas desarmonias sociais que afetaram os determinantes e condicionantes de saúde, como modos de vida, trabalho, habitação, ambiente, lazer, acesso a bens e serviços. Assim, pôde-se dizer que o racismo é o principal fator que afeta os determinantes de saúde na população negra, por refletir desfavoravelmente sobre todas as necessidades que fazem parte da abrangência do conceito sobre saúde (BRASIL, 2017).

Apesar deste estudo ter sido realizado em 2018, outras pesquisas recentes evidenciam uma abordagem semelhante quanto ao racismo institucional sofrido pelos portadores dessa patologia, seus familiares e/ou cuidadores. Segundo Marques T, et al. (2019), os transtornos falciformes mostram uma real vulnerabilidade social, ao acometer populações menos favorecidas, ocupando a base da pirâmide social e apresentando os piores indicadores educacionais, socioeconômicos e epidemiológicos. Como exemplo, têm-se as maiores taxas de desemprego, menor participação no produto interno bruto (PIB), pior acesso à saúde.

Gráfico 1 - Tendência de variação da mortalidade por transtornos falciformes nas macrorregiões de saúde no estado da Bahia no período de 2012 a 2016.

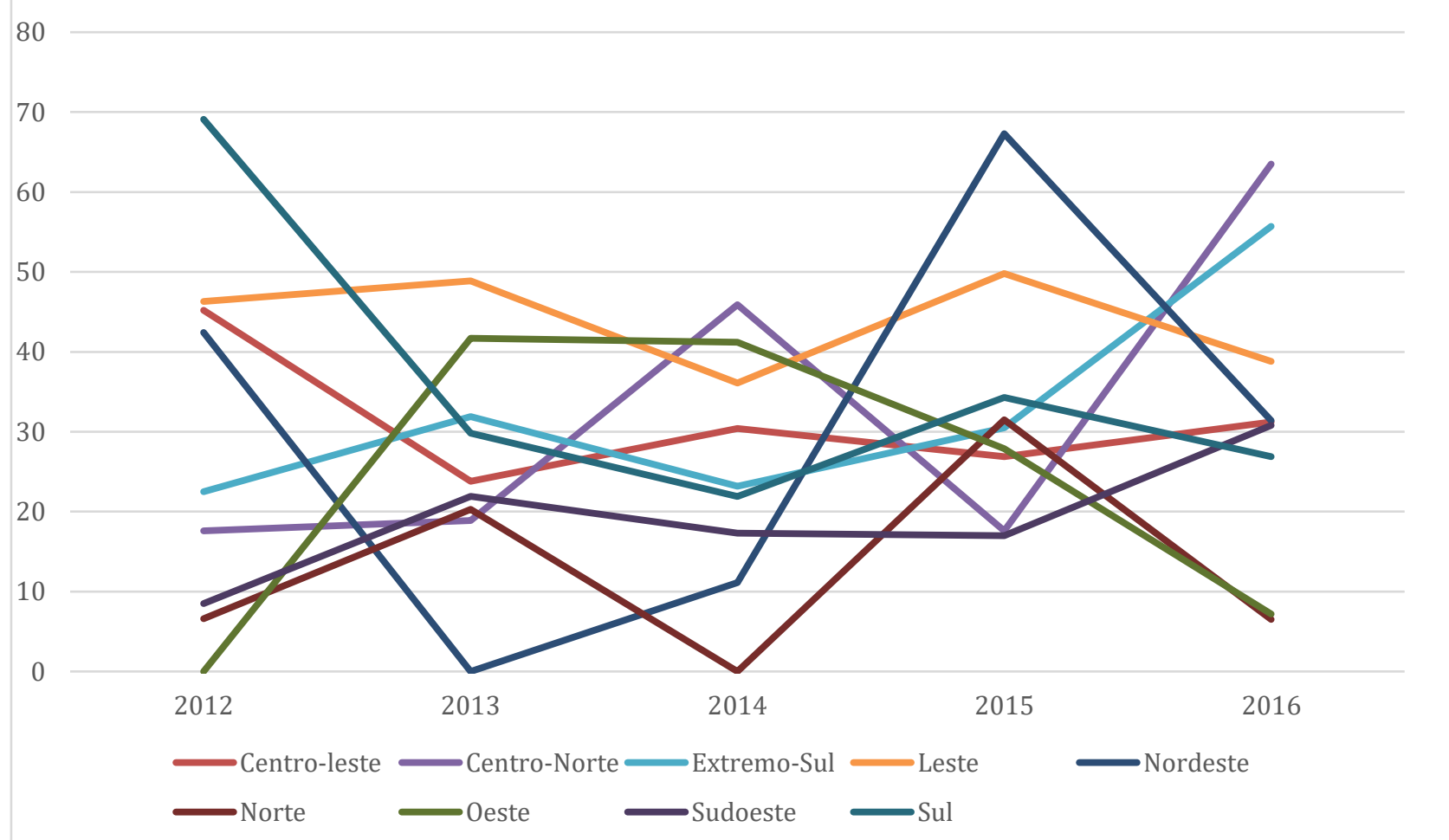

Fonte: Santana DM et al., 2020. 
Tabela 1 - Taxa de mortalidade por transtornos falciformes por ano segundo as macrorregiões de saúde no estado da Bahia no período de 2012 a 2016.

\begin{tabular}{llllll}
\hline \multirow{1}{*}{ Região } & \multicolumn{5}{c}{ Ano } \\
\cline { 2 - 6 } & $\mathbf{2 0 1 2}$ & $\mathbf{2 0 1 3}$ & $\mathbf{2 0 1 4}$ & $\mathbf{2 0 1 5}$ & $\mathbf{2 0 1 6}$ \\
\hline Centro-leste & 45,2 & 23,8 & 30,4 & 26,9 & 31,2 \\
Centro-Norte & 17,6 & 18,9 & 45,9 & 17,6 & 63.5 \\
Extremo-Sul & 22,5 & 31,9 & 23,2 & 30,5 & 55,7 \\
Leste & 46,3 & 48,9 & 36,1 & 49,8 & 38,8 \\
Nordeste & 42,4 & --- & 11,1 & 67,3 & 31,4 \\
Norte & 6,6 & 20,3 & -1, & 31,5 & 6,5 \\
Oeste & ---- & 41,7 & 41,2 & 27,9 & 7,2 \\
Sudoeste & 8,5 & 21,9 & 17,3 & 17,0 & 30,8 \\
Sul & 69,1 & 29,8 & 21,9 & 34,3 & 26,9 \\
\hline
\end{tabular}

Fonte: Santana DM et al., 2020. Dados coletados do Sistema de Informação sobre Mortalidade (SIM). (2018).

Na variável faixa etária, entre os anos de 2012 a 2016, constatou-se uma maior quantidade de óbitos em 2015, com quantitativo de 73 óbitos com o coeficiente de mortalidade de 35,7 para 100.000 nascidos vivos, que ocorreram em indivíduos no estudo da população com idade entre 0 a 79 anos, mais frequente entre as idades de 30 a 39 anos, com o quantitativo de 22 casos. Em compensação, no ano de 2016, houve um menor número de mortalidade por transtorno falciforme, com apenas 13 casos de óbitos, com idade entre 40 a 49 anos, em indivíduos de 1 a 69 anos. Nota-se que o número de óbitos predominou entre jovens e adultos, provavelmente a recorrentes crises álgicas, vaso-oclusivas, úlceras, AVE, infecções, icterícia, priapismo e sequestro esplênico, os quais contribuem para diminuição da expectativa de vida dos portadores.

A redução nos casos de mortalidade em 2016 evidenciou o quantitativo de 65 óbitos, com o coeficiente de mortalidade de 32,7 por 100.000 nascidos vivos, o que sugere possíveis condições determinantes, como subnotificações dos casos da doença, redução na cobertura assistencial hospitalar, implementação de programas de saúde direcionados aos portadores de DF e aumento na assistência dos hemocentros nas terapias transfusionais.

Pesquisas recentes apontam o aumento da letalidade da DF mostrando grande impacto social da patologia, evidenciando a necessidade de uma atenção direcionada aos portadores da doença. Faz-se necessária uma mobilização do Ministério da Saúde para implantar uma assistência voltada exclusivamente para o portador do transtorno falciforme, construindo metas futuras que possam viabilizar políticas públicas eficientes, como a Triagem Neonatal e todos os programas vinculados ao Ministério da Saúde (RAMOS JT, et al., 2015).

A doença falciforme é uma enfermidade genética que acomete, tanto o gênero masculino quanto o feminino. Nesse estudo houve maior número de óbitos no sexo masculino, correspondendo a um total de 73 óbitos no ano de 2012, mostrando o coeficiente de mortalidade 35,2 por 100.000 nascidos vivos, sendo que nesse ano ocorreram 44 casos de óbitos no sexo masculino.

Os estudos mencionam a dificuldade dos homens frequentarem voluntariamente o serviço de saúde, principalmente, aqueles direcionados à promoção e prevenção de doenças, pois não conseguem se expressar, colocando-se em situação de risco, já que procuram os serviços de saúde apenas quando não suportam os sinais e sintomas. Para esse grupo, há uma forte influência da cultura machista, havendo demonstração de fragilidade, quando relatam sua dor ou por medo e receio de afetar o papel provedor do homem. Contudo, torna-se essencial um acolhimento com mais sensibilidade pelos profissionais de saúde para as questões que envolvem concepções de gênero, incluindo as necessidades dos homens enquanto usuários do Sistema Único de Saúde (SUS) (GOMES LMX, et al., 2014).

De acordo com Pompeo CM, et al. (2020), um estudo recente realizado sobre os fatores de risco em pacientes com doença falciforme evidenciou uma maior taxa de mortalidade no sexo masculino. Apesar desse achado ser mais recente, em 2020, Pompeo CM, et al. (2020) não evidenciam uma possível relação entre altas taxas de mortalidade por transtornos falciformes e o sexo masculino, afirmando que outros estudos também constataram altas taxas de mortalidade entre as mulheres, principalmente, na fase adulta. Para esses autores, não existe relação entre os transtornos falciformes e o sexo dos indivíduos. 
Em relação a variável estado civil, houve uma maior mortalidade por transtorno falciforme em indivíduos solteiros, correspondendo ao total de 40 óbitos no ano de 2015. Acredita-se que os indivíduos solteiros tenham uma menor percepção do seu estado de saúde, apresentando maior propensão ao óbito. Em sua maioria, os jovens e adultos solteiros priorizam outros interesses pessoais, podendo ocasionar uma procrastinação do autocuidado, permitindo um comportamento de risco. As manifestações clínicas da doença falciforme podem prejudicar a qualidade de vida desses indivíduos solteiros, podendo torná-los inativos, afetando sua perspectiva de vida futura (CUNHA JHS, et al., 2017).

Os transtornos falciformes podem comprometer a capacidade dos indivíduos de enfrentarem seus problemas, bem como o potencial para se tornarem seres produtivos, causando redução na sua qualidade de vida (QV). Sendo assim, é essencial entender qual a percepção dos portadores de transtornos falciformes sobre sua qualidade de vida, para que sejam planejadas e estabelecidas condutas adequadas pelos profissionais de saúde, em especial, o enfermeiro (FREITAS SLF, et al., 2018).

Para a variável escolaridade, no ano de 2013, ocorreu o maior número de óbitos, havendo 21 casos. O tempo de escolaridade foi entre 8 e 11 anos, correspondendo ao ensino Fundamental II e $2^{\circ}$ ano do Ensino Médio. No ano de 2016 houve 65 casos de óbitos com o coeficiente de mortalidade de 32,7 para cada 100.000 nascidos vivos, em indivíduos com escolaridade entre 4 a 7 anos de estudos, compreendendo o ensino Fundamental I e II incompletos e correspondendo a 17 óbitos. Nesse mesmo estudo, o grupo Ignorados, sugestivos de analfabetos, indigentes, indivíduos mal avaliados pelos profissionais de saúde, etc. apresentou elevado número de óbitos. Nos anos 2012 e 2013 foram 26 casos ignorados. Acredita-se que nesse grupo houve falha no preenchimento dos formulários informatizados, o que pode ter comprometido a veracidade dos dados.

Em uma pesquisa realizada por Ramos JT, et al. (2017), que avaliou a mortalidade infantil por doença falciforme, na Bahia, entre 2008 e 2013, verificou-se elevada taxa de letalidade e prevalência dessa patologia em lactentes menores de um ano. Os autores relataram que em estudos baseados em dados secundários, oriundos dos sistemas de informação, há um risco de subnotificações das informações referentes aos óbitos das crianças por transtornos falciformes. Isso é muito preocupante, considerando que a identificação precoce dos transtornos falciformes, com a adequada introdução das intervenções terapêuticas, poderá reduzir as complicações e facilitar a adaptação à doença.

O não preenchimento do SIM (Sistema de Informação da Mortalidade) pode afetar diretamente a coleta de dados sobre as taxas de mortalidade, sendo fundamental a atualização do sistema de informação em saúde, o qual contribui com informações fidedignas para embasamento de futuros estudos científicos. Esses dados são necessários para pesquisas sociodemográficas e epidemiológicas, contribuindo assim para a elaboração e implementação de políticas públicas direcionadas à população negra (RAMOS JT, et al., 2015).

Indivíduos não alfabetizados tendem a ter uma menor adesão ao tratamento e à aceitação da doença, em decorrência da falta de conhecimentos, resistência à informação, medo do preconceito, comprometendo a busca pela assistência especializada, podendo gerar complicações no quadro clínico. Esses indivíduos apresentam crenças e tabus oriundos de hábitos, costumes, os quais podem comprometer a compreensão dos transtornos falciformes.

A escolaridade traz inúmeros benefícios para melhor perspectiva de vida e prognóstico da doença. A escola representa um local propício a informações, esclarecimentos, educação, saúde, interferindo diretamente na qualidade de vida do indivíduo, desenvolvendo melhor autoestima. A educação com propriedade traz conhecimentos, habilidades para melhor aceitação da doença, bem como o tratamento (CRUZ SV, et al., 2016).

De acordo com a variável óbitos por ocorrência, verificou-se maior índice de óbitos no ano de 2015, nos hospitais, com 73 casos. Contudo, no ano 2016 houve 65 casos de óbitos por transtorno falciforme. Pôde-se perceber que as mortes em âmbito hospitalar ocorreram através de possíveis complicações da doença, ocasionando a hospitalização dos portadores, com prováveis manifestações de crises álgicas e síndrome torácica aguda, sepse, problemas cardiorrespiratórios, insuficiência renal, dentre outras complicações (RAMOS JT, et al., 2015). 
Os portadores dessa doença podem apresentar sinais e sintomas passíveis de serem controlados em ambulatório, mas podem desenvolver complicações que requerem internamento. A adesão do paciente e seus familiares ao tratamento e às formas de prevenção é importante, mas a assistência em enfermagem precisa considerar todos os aspectos, incluindo o físico, emocional, cultural e social dos indivíduos com transtornos falciformes e que as condutas de enfermagem sejam registradas de forma clara e direcionadas (CARVALHO EMMS, et al., 2015).

\section{CONCLUSÃO}

O estudo evidenciou que a maioria dos óbitos ocorreu no ambiente hospitalar, por adultos jovens e negros, constatando um possível comprometimento do estado de saúde das vítimas, decorrente das complicações graves dos transtornos falciformes, podendo sugerir possível ausência de diagnóstico precoce e assistência adequada pelos profissionais de saúde, desde a atenção básica, até a média e alta complexidade. Torna-se indiscutível a necessidade de acolhimento adequado e direcionado aos portadores, já que essa patologia é comprometida pelo racismo institucional e estrutural. Os transtornos falciformes representam uma patologia crônica e suas complicações são responsáveis por aumentar as taxas de morbidade e mortalidade. Apesar da escassez de pesquisas no Brasil, sobre a mortalidade por transtornos falciformes, esse estudo foi essencial para demonstrar parte do perfil da população de óbitos, além de ter identificado a região Leste com maior taxa de mortalidade no estado, abrigando a maior população de afrodescendentes. O estudo poderá contribuir para reformular e direcionar políticas públicas e recursos para a adequada assistência aos portadores dos transtornos e do traço falciforme.

\section{REFERÊNCIAS}

1. BRASIL. Manual do Ministério da Saúde. 2014

$\begin{array}{llllll} & & & \\ \text { 2. https://bvsms.saude.gov.br/bvs/publicacoes/doenca_falciforme_deve_saber_sobre_heranca.pdf } & & \text { Disponível } & \text { em: }\end{array}$

3. BRASIL. Manual do Ministério da Saúde. 2015. Disponível em: http://biblioteca.cofen.gov.br/wpcontent/uploads/2017/09/doenca_falciforme_diretrizes_basicas_linha_cuidado.pdf

4. BRASIL. Manual do Ministério da - Saúde. 2017. Disponível em: http://bvsms.saude.gov.br/bvs/publicacoes/politica_nacional_saude_populacao_negra_3d.pdf.

5. BRASIL. Manual do Ministério da Saúde. 2013. Disponível em: https://www.nupad.medicina.ufmg.br/wpcontent/uploads/2016/12/Manual-Doenca-Falciforme-Ulceras-tratamento-e-prevencao.pdf

6. CARVALHO EMMS, et al. Doença falciforme nas pesquisas em enfermagem: uma revisão integrativa, Brasil. Revista Baiana de Enfermagem, 2015; 29: 86-93.

7. CRUZ SV, et al. Avaliação da qualidade de vida em pacientes adultos com anemia falciforme no norte de Minas GeraisBrasil, Brasil. Rev. Med. Minas Gerais, 2016; 26: S23-S30.

8. CUNHA JHS, et al. Papéis ocupacionais de indivíduos com anemia falciforme, Brasil. Rev Ter Ocup Univ São Paulo, 2017; 28: $230-8$.

9. FIGUEIREDO AKB, et al. Anemia falciforme: abordagem diagnóstica laboratorial, Brasil. Rev. Ciênc. Saúde Nova Esperança, 2014; 12: 96-103.

10. FIGUEIREDO W. Assistência à saúde dos homens: um desafio para os serviços de atenção primária. Ciênc. Saúde Coletiva, 2014; 10(1): 105-109.

11. FIGUEIRÓ AVM, RIBEIRO RLR. Vivência do preconceito racial e de classe na doença falciforme. Saúde. Soc, 2017; 26(1):88-99.

12. FREITAS SLF, et al. Qualidade de vida em adultos com doença falciforme: revisão integrativa da literatura, Brasil. Revista Brasileira de Enfermagem, 2018; 71: 195-205.

13. GOIS EC, et al. Mortalidade materna na Bahia no período de 2012 a 2016, Brasil. Revista Eletrônica Acervo Saúde, 2019; 18: 1-8.

14. GOMES LMX, et al. Acesso e assistência à pessoa com anemia falciforme na atenção primária, Brasil. Acta Paul Enferm, 2014; $27: 348-55$.

15. LAGES SRC, et al. O preconceito racial como determinante social da saúde - a invisibilidade da anemia falciforme, Brasil. Revista Interinstitucional de Psicologia, 2017; 10: 109-122.

16. MARQUES T, et al. Perfil clínico e assistencial de crianças e adolescentes com doença falciforme no nordeste brasileiro, Brasil. Rev. Bras. Saúde Mater. Infant., 2019; 19: 889-896.

17. OLIVEIRA AS, CARVALHO AR. A desigualdade racial do Brasil: o racismo estrutural e o determinismo social. Revista Jurídica Direito, Sociedade e Justiça/RJDSJ, $2017 ; 5$ (1): 228/230.

18. POMPEO CM, et al. Fatores de risco para mortalidade em pacientes com doença falciforme: uma revisão integrativa, Brasil. Escola Anna Nery, 2020; 24: 1-11.

19. RAMOS JT, et al. Mortalidade infantil por doença falciforme na Bahia: um estudo epidemiológico, Brasil. Rev. Saúde Col., 2017; 7: 36-39.

20. RAMOS JT, et al. Mortalidade por doença falciforme em estado do nordeste brasileiro, Brasil. R. Enferm. Cent. O. Min., 2015; 5: 1604-1612.

21. SILVA NCH, et al. Principais técnicas para o diagnóstico da anemia falciforme: uma revisão de literatura, Brasil. Rev. Ciências Biológicas e de Saúde Unit, 2017; 3: 33-46. 